\title{
Existence of log-phase Escherichia coli persisters and lasting memory of a starvation pulse
}

\author{
Mikkel Skjoldan Svenningsen ${ }^{1}$, Sine Lo Svenningsen², Michael Askvad Sørensen² (1), Namiko Mitarai ${ }^{1}$ (D)
}

\begin{abstract}
The vast majority of a bacterial population is killed when treated with a lethal concentration of antibiotics. The time scale of this killing is often comparable with the bacterial generation time before the addition of antibiotics. Yet, a small subpopulation typically survives for an extended period. However, the long-term killing dynamics of bacterial cells has not been fully quantified even in well-controlled laboratory conditions. We constructed a week-long killing assay and followed the survival fraction of Escherichia coli K12 exposed to a high concentration of ciprofloxacin. We found that long-term survivors were formed during exponential growth, with some cells surviving at least $7 \mathrm{~d}$. The long-term dynamics contained at least three time scales, which greatly enhances predictions of the population survival time compared with the biphasic extrapolation from the short-term behavior. Furthermore, we observed a long memory effect of a brief starvation pulse, which was dependent on the (p)ppGpp synthase relA. Specifically, $1 \mathrm{~h}$ of carbon starvation before antibiotics exposure increased the surviving fraction by nearly 100fold even after $4 \mathrm{~d}$ of ciprofloxacin treatment.
\end{abstract}

DOI 10.26508/Isa.202101076 | Received 26 March 2021 | Revised 4 November 2021 | Accepted 4 November 2021 | Published online 18 November 2021

\section{Introduction}

Bacterial populations are quickly decimated during a typical antibiotics assault. Within a few generation times, the far majority of cells will be dead. However, it is typically recommended to use extended durations of treatment, ranging from several days to months, prolonging the exposure time of bacterial pathogens to the antibiotic (1). The WHO is now considering the benefits of shortening the duration of antibiotics administration while still keeping the treatment effective because of concerns of increasing antibiotic resistance occurring as a consequence of increased exposure (1). To find the optimal treatment duration, one needs to understand the killing dynamics of bacteria when exposed to antibiotics, especially the bacterial cells surviving for longer times. The long-term survivors are typically referred to as persister cells, a subgroup of cells that survive antibiotics for an extended period compared to the average of the population but have not acquired mutations that make them resistant to the antibiotic $(2,3)$. Most research on persister cells is performed within the well-defined conditions of the laboratory, but despite these strongly simplified conditions, and more than $70 \mathrm{yr}$ of research, laboratory persisters are still far from understood $(4,5)$.

One pending question is whether and how much persisters form spontaneously during the exponential growth phase. Such persisters are called type-II (3) or spontaneous (4) persisters. It was repeatedly shown that stress-triggered (or type I) persisters are formed in high numbers during the stationary phase, but research on spontaneous persister formation during the exponential phase is sparse (4). The research has mostly been confounded by a lack of careful attention to the presence of stationary-phase cells carried over from the starter cultures, which artificially elevated the persister fraction of exponential cultures $(4,5,6)$. One carefully executed study showed that no Escherichia coli persister cells were formed during fast exponential growth in rich medium (7), whereas other studies merely showed reduced levels during exponential growth $(3,5)$. A benefit of analysing the exponential growth phase is the well-defined physiology of this state $(8,9)$. This makes it possible to vary the growth physiology in a controlled manner, especially by varying the growth rate through culturing bacteria in media of different nutrient quality. It was previously shown that the bacterial growth rate strongly correlates with the death rate during the initial period of killing with $\beta$-lactams $(10,11,12)$. This poses the additional questions of how the growth rate at the time of antibiotics exposure affects the short- and long-term killing dynamics.

The current standard for persister identification at the population level is that the killing curve is at least biphasic, where two time scales are identified in the time-kill curves (4). Persisters are identified as the subpopulation with a second, slower killing rate than the rapid death rate of the primary population. If only two time scales are present in the killing dynamics, the population survival time can be extrapolated from the second slow killing rate. Notably, the presence of more than two phases has been demonstrated previously in a few studies $(3,13,14,15)$. These observations motivate the importance of studying the long-term survival of the antibiotics-tolerant subpopulation, which may not agree with extrapolation from short-term survival. However, most in vitro

${ }^{1}$ The Niels Bohr Institute, University of Copenhagen, Copenhagen, Denmark ${ }^{2}$ The Department of Biology, University of Copenhagen, Copenhagen, Denmark 
laboratory research on persisters of fast-growing bacteria as $E$. coli is carried out for $3-5 \mathrm{~h}(5,7,16)$, although some studies increase the exposure time to $24-50 \mathrm{~h}(2,3,15,17)$. Investigation of long-term survival beyond the typical 5 -h persister assay might reveal new insights into bacterial killing dynamics that are relevant for the week-long antibiotics treatment of bacterial infections recommended by the WHO (1).

Last, the molecular mechanism(s) of persister formation is still unknown $(12,18)$. Many intracellular components have been proposed to play a role $(16,19,20,21,22,23)$, but so far no single mechanism convincingly explains persister formation. In fact, bacterial persistence presents as a very complex and diverse problem, where the survival fraction could be composed of different subpopulations.

Despite the complexity of persistence, it has been established that stationary-phase cultures contain a greater persister fraction than exponentially growing cultures (5). Stationary-phase bacteria may refer to bacteria in a multitude of different physiological states but is typically associated with starvation stress (24). Furthermore, the second messenger ( $p$ )ppGpp, which accumulates during starvation responses, was frequently shown to correlate positively with persistence formation $(5,17,21,25,26)$. Hence, it is critical for persistence research to understand the degree to which (p)ppGpp levels affect persistence, and under which circumstances.

The present study investigates persistence in the balanced exponential growth phase where (p)ppGpp levels are relatively low and correlate inversely with the growth rate. It deals with whether E. coli forms spontaneous persisters in the exponential phase, their dependence on the growth rate, how long they survive and how their formation relates to (p)ppGpp levels. We followed the longterm survival of E. coli K12 populations exposed to a lethal concentration of ciprofloxacin for 1 wk. The growth rate of the E. coli population at the time of antibiotics exposure was varied using growth medium with either of two different carbon sources. In addition, a knockout strain was constructed in the wild-type background, removing the gene relA and, thus, introducing a ( $p$ ) ppGpp synthesis deficiency. Furthermore, we compared the killing dynamics with and without a short carbon starvation period immediately before the killing assay. The starvation pulse had a considerable influence on persister formation. This triggered persistence had a very long memory effect on the survival of the population.

\section{Results}

\section{Long-term persister assay of exponentially growing cells}

First, we investigated whether long-term persister cells form during exponential growth in glucose minimal medium. We treated balanced cultures of $E$. coli K-12 with ciprofloxacin and monitored the killing dynamics for 1 wk of antibiotics treatment. Balanced growth was obtained by culturing the cells for more than 20 doubling times in the target medium at $37^{\circ} \mathrm{C}$, keeping the cell density of the culture below an $\mathrm{OD}_{436}$ of 0.3 by repeated back dilutions. In total, at least $10^{9}$-fold dilutions were performed during the exponential phase to ensure that the reported persister cell numbers are well above the possible number of carry-over cells from stationary phase. Cultures were then treated for a week with $10 \mu \mathrm{g} / \mathrm{ml}$ ciprofloxacin and their killing dynamics were monitored by repeated platings of culture aliquots on antibiotics-free growth medium (See the Materials and Methods section for details).

E. coli persisters were formed during exponential balanced growth in glucose minimal medium, as seen in Fig 1. There was a fast initial killing at a rate of about $1 / 0.3\left(h^{-1}\right)$, with a slower killing rate already after $2 \mathrm{~h}$. When we fit a biphasic curve (summation of two exponential functions) to the data up to $7 \mathrm{~h}$, the second phase of killing is at a rate of about $1 / 2.3\left(\mathrm{~h}^{-1}\right)$, shown by a brown line in the inset in Fig 1. However, for longer times, this fit significantly underestimates the survival time of the bacterial population (Fig 1). In other words, there is an even slower phase of killing, extending from $7 \mathrm{~h}$ to $4 \mathrm{~d}$. Last, from day 5 to day 7 , the remaining cells were killed at a very slow rate; however, this part of the data is less reliable because of the small numbers of recovered colonies.

To identify the various phases in a quantitative manner, the data were fitted with a sum of exponentials, and the appropriate number of exponentials was chosen with a $x^{2}$-test (see the Materials and Methods section). The double exponential, which corresponds to the biphasic killing dynamics, was rejected as a good fit by the test. Instead, a fit using the triple exponential functions was accepted. The best fit obtained was the first phase of killing at a rate $1 / 0.3\left(\mathrm{~h}^{-1}\right)$ (a cyan dashed line), the second phase of killing at a rate of $1 / 8\left(\mathrm{~h}^{-1}\right)$ (a green dashed line), and the third phase of killing at a rate of $1 / 57$ $\left(\mathrm{h}^{-1}\right)$ (a blue dashed line). The long-term killing dynamics thus had more than two time scales, which is only apparent after several days of measurement.

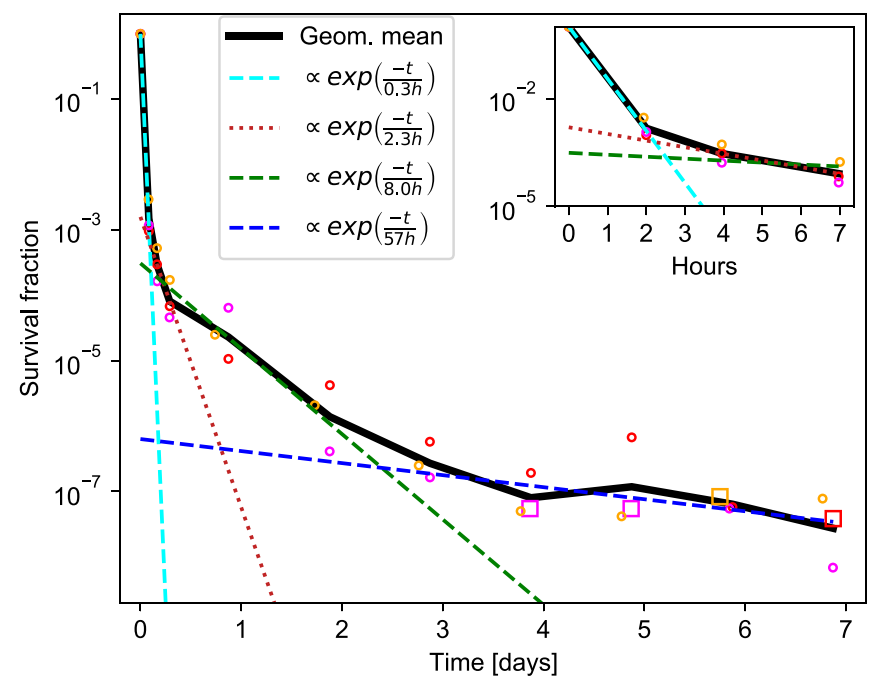

Figure 1. Killing dynamics of exponential-phase Escherichia coli persisters. Killing dynamics for exponential-phase persisters. The bacteria were grown in glucose minimal medium. All three biological replicates are shown for each data point (circle). When zero colonies are recovered, the detection limit is shown (square, see the Materials and Methods section for details). The black line is the geometrical mean. Each separate phase of a triphasic fit is shown, respectively, as cyan, green, and blue dashed lines. The second phase of a biphasic fit to the first $7 \mathrm{~h}$ is shown as a brown dotted line. 
The exponential-phase growth rate determines many aspects of bacterial physiology, including the macromolecular composition (8, 27). The growth rate at the time of antibiotics exposure has previously been linked to short-term survival of antibiotics $(10,11)$ and could also affect long-term survival. For that reason, the long- term killing assay was repeated with glycerol as the carbon source, which strongly affected the wild-type growth rate. In glucose minimal medium, the wild-type doubling time was $50 \pm 1.4$ min, whereas it was $106 \pm 3.0 \mathrm{~min}$ in glycerol minimal medium. This difference had an impact on the initial phase of killing for up to $7 \mathrm{~h}$,

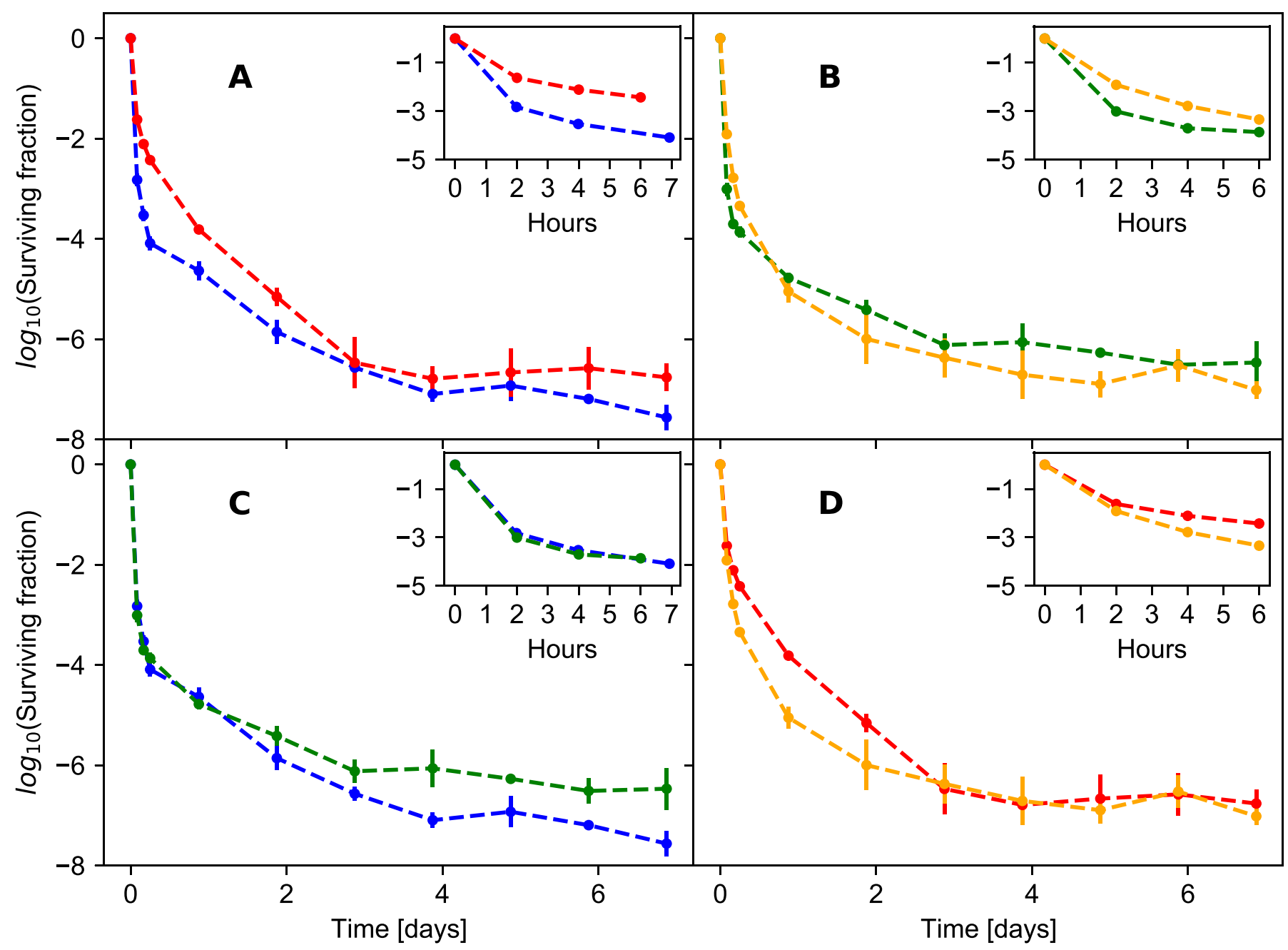

- - WT glucose

$-\uparrow-\quad$ relA glucose

$-\uparrow-$ WT glycerol

$-\uparrow-\quad$ relA glycerol

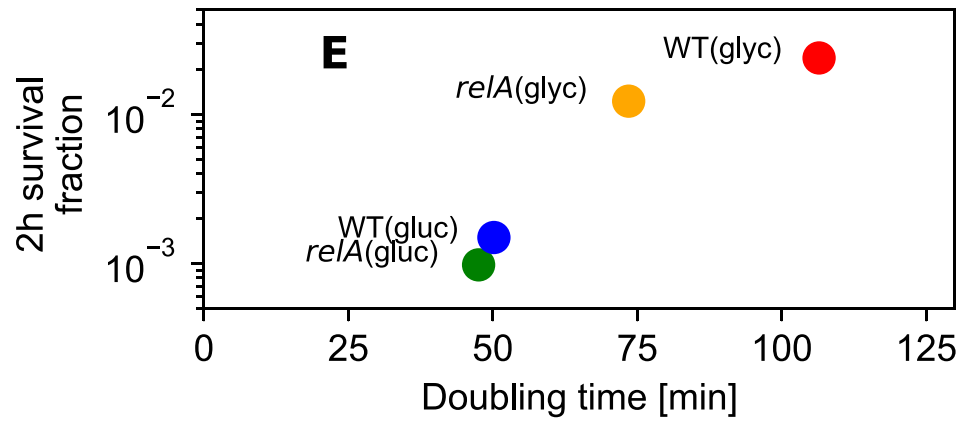

Figure 2. Killing dynamics in different media with and without the relA gene.

Each line represents a geometric average of three biological replicates. (A) The wildtype killing dynamics in either glycerol or glucose minimal medium. (B) The $\Delta$ relA killing dynamics in either glycerol or glucose minimal medium. (C) Killing dynamics in glucose minimal medium for comparison between the wildtype and the $\Delta$ relA strain. There was no statistically significant difference between the wildtype survivors and the $\Delta$ relA strain survivors at any of the timepoints (see the Materials and Methods section). (D) Killing dynamics in glycerol minimal medium for comparison between the wildtype and the $\Delta$ relA strain. (A, B, C, D) Log-log plot of the data in (A, B, C, D) is available in Fig S1. (E) Survival fraction after $2 \mathrm{~h}$ of ciprofloxacin treatment plotted against the growth rate before antibiotics treatment. 
showing slower killing and a higher survivor fraction in glycerol (Fig $2 \mathrm{~A}$ inset).

However, the long-term killing curve in glycerol minimal medium was not merely a decelerated version of the killing curve in glucose minimal medium. Although significantly more cells survive in glycerol than in glucose for up to a day (Fig 2A), the survival curve in glycerol medium from 7 to $21 \mathrm{~h}$ had a steeper slope than in glucose medium, resulting in comparable surviving fractions after $2-3 \mathrm{~d}$. In fact, in two of the three biological replicates of wild-type cultures grown in glycerol, almost no survivors were observed after $3 \mathrm{~d}$ of killing (see Fig S2), supporting further that the survival in glycerol minimal medium is not more than that in glucose minimal medium after $3 \mathrm{~d}$.

Overall, the wild-type killing dynamics in glycerol had more than two time scales, with a best fit of four separate phases of killing (Fig S3).

\section{Deletion of relA affects the killing dynamics}

In glycerol minimal medium, the steady state (p)ppGpp level of the wild-type strain is higher than in glucose minimal medium (28). Because the (p)ppGpp level has been frequently associated with persister formation, we next aimed at investigating the effect of the initial (p)ppGpp level on long-term survival. E. coli encodes the primary ( $p$ )ppGpp synthetase RelA and the secondary (p)ppGpp synthetase SpoT, the latter of which is bifunctional as a (p)ppGpp hydrolase. The nutrient-dependent steady-state growth rates are inversely related to the concentrations of (p)ppGpp, both for relA ${ }^{+}$ and rel $A^{-}$strains (28), and for many carbon sources, the growth rates and (p)ppGpp levels of rel$A^{*} /$ rel $A^{-}$strain pairs are indistinguishable because of (p)ppGpp synthesis by SpoT. However, in lowenergy carbon sources such as glycerol or acetate, SpoT produces insufficient ( $p$ )ppgpp to reduce the growth rate when RelA is missing, leading to an enhanced growth rate of the RelA mutant strain relative to the wild type (28). We constructed a $\triangle$ relA mutant to clarify the role of (p)ppGpp in the killing dynamics.

As expected, the difference between the growth rate in glucose and in glycerol minimal medium was smaller for the $\Delta$ relA strain than the wild type, with a doubling time of $47 \pm 1.5 \mathrm{~min}$ in glucose minimal medium and $74 \pm 1.6 \mathrm{~min}$ in glycerol minimal medium. The survival after $2 \mathrm{~h}$ is positively correlated with the doubling time (Fig $2 \mathrm{E}$ ), consistent with the previous observations that the initial killing rate decreases with the doubling time (11). The short-term (up to $4 \mathrm{~h}$ ) survival under antibiotics exposure was also correlated with the growth rate in the $\triangle$ relA strain (Fig 2 inset).

Like wild-type cells, the $\triangle$ relA mutant formed long-term survivors in both glucose and glycerol minimal medium with more than two phases of killing (Fig 2B, see the Materials and Methods section for statistical analysis). In glucose, the two strains grew at similar rates, and the early killing dynamics of the $\triangle r e l A$ mutant were very similar to that of the wild type (Fig $2 \mathrm{C}$ ). In glycerol, the faster growing $\Delta$ relA mutant showed a significantly lower level of persisters than the wild type in the initial phase of killing up to $1 \mathrm{~d}$ (Figs 2D and S1), indicating the importance of growth rate, or ppGpp level, for persister formation in this phase. Interestingly, the long-term survival of the $\Delta$ relA mutant and wild type were comparable at later times (after $3 \mathrm{~d}$ ) in glycerol medium, and somewhat higher than the wild-type strain in glucose medium. Thus, survival in the long term is not simply dependent on the population growth rate at the time of antibiotics exposure.

\section{A starvation pulse before the antibiotic application affects the long-term persistence of wild-type cells}

A sudden downshift of the carbon source is known to give a spike of the (p)ppGpp level in the wild-type strain just after the downshift, whereas the spike is significantly lower in a RelA strain (29).

We then wondered if a short pulse of carbon source starvation to the exponentially growing cells before the killing assay would give a quantifiable difference in the long-term persistence between the wild-type strain and the $\Delta$ relA strain. If the starvation pulse increases the persisters, it would be considered as triggered persistence, and the current study would allow us to quantify how long such triggered persisters last.

To test this, part of the cultures in balanced growth were filtered into growth medium without a carbon source and starved for $1 \mathrm{~h}$, before the carbon source was replenished (Fig 3A). Fig 3B shows that the 1-h starvation pulse resulted in a quick rise of the ppGpp level peaking at about 15 min after the downshift for the wildtype strain in glucose medium, whereas only a mild increase in the ppGpp level was seen in the $\Delta$ relA strain. Antibiotics were added simultaneously with the carbon source replenishment (Fig 3A, see the Materials and Methods section for details). Remarkably, the short carbon starvation before the addition of antibiotics had longterm effects on the killing dynamics. This was especially visible for the wild-type strain grown in glucose minimal medium, where the brief starvation period reproducibly resulted in almost 100-fold more persisters for up to $4 \mathrm{~d}$ (Fig 4A). The difference was abolished by removing relA, as seen in Fig 4B; the $\triangle$ relA strain only exhibited increased survival for the first $6 \mathrm{~h}$ after starvation. As such, the longterm memory of the starvation pulse is seemingly a relA-dependent effect. However, the $\triangle$ relA strain in the steady-state growth in glucose had more long-term survivors than the wild-type strain, and the downshift brought the wild-type strain survival fraction to a level similar to the $\triangle$ relA strain.

The effect of the downshift was smaller in the glycerol medium (Fig 4C and D). In the wild-type strain, the average persister level with downshift was higher up to $4 \mathrm{~d}$, but the statistical significance of the difference was confirmed only up to $7 \mathrm{~h}$ because of the larger data scatter for later time points (Fig 4C). The effect of downshift disappeared faster in the $\Delta$ relA strain already after $2 \mathrm{~d}$ (Fig 4D).

\section{Discussion}

We expanded the understanding of bacterial killing dynamics with a long-term persister assay. The use of minimal medium facilitated the formation of long-term persisters during exponential growth, in contrast to growth and killing in rich medium (7). Spontaneous persisters were observed during the exponential growth phase, both in glucose and glycerol minimal medium, and in some cases, they survived at least $1 \mathrm{wk}$. This long-term survival does not require relA, although the residual (p)ppGpp synthesized by SpoT is likely 
A

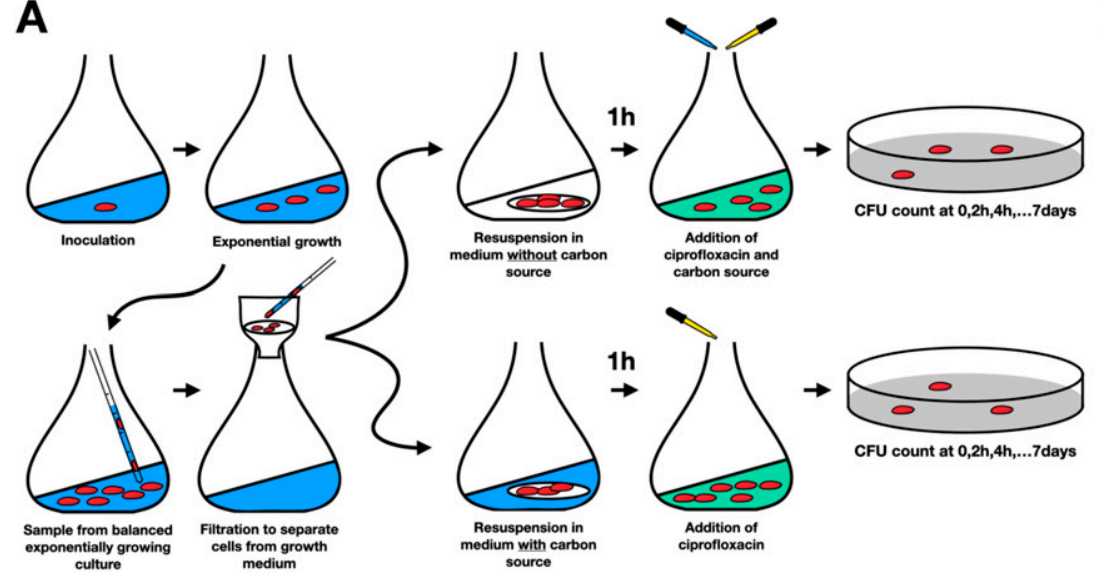

B

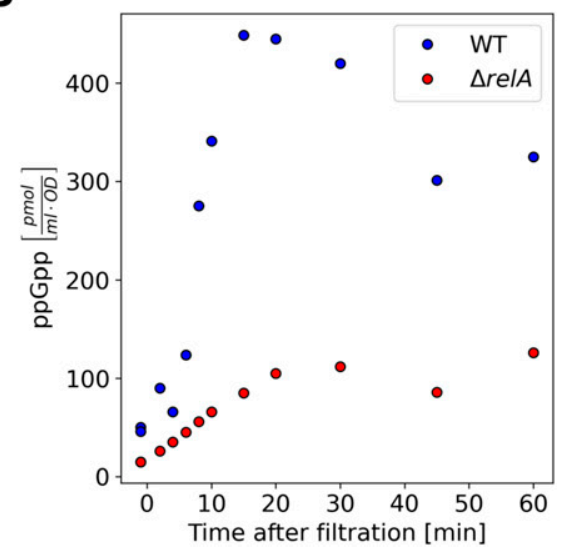

Figure 3. The experimental setup for the long-term persistence assay.

(A) Cells were in balanced exponential growth before the killing assay. A part of the culture was filtered and then resuspended in medium with or without the carbon source, and incubated for $1 \mathrm{~h}$. After $1 \mathrm{~h}$, the ciprofloxacin was added to both of the samples, and at the same time the carbon source was added to the culture that has been starved for $1 \mathrm{~h}$. The first sample was taken just before the additions. After that, samples were taken at 2, 4, 6/7,21+24 $\mathrm{n}$ hours after the antibiotic addition for $n \in[0 ; 6]$. The samples were washed and plated on agar plates containing the target medium. (B) The 1-h time course of ppGpp level for the culture grown in the glucose medium, filtered, and resuspended in fresh medium without carbon source. The time zero is the time of the resuspension. The wild type (blue circles) shows clear peak around 15 min after the resuspension. $\triangle$ relA strain shows only mild increase in the ppGpp level. The phospholmager scan of TLC plates used to quantify ppGpp levels is shown in Fig S4

necessary. In fact, there is an increase in long-term survival of the $\Delta$ relA mutant in glucose.

We have shown that a 1-h starvation pulse before the addition of the antibiotic affects long-term survival. The finding that a short starvation pulse gives a long-term effect is consistent with a previous study of a temporal nitrogen downshift before antibiotics treatment, which was shown to elevate the persister level at $24 \mathrm{~h}$ in a relA-dependent manner (30). Also, a few other studies have previously shown that triggered persistence can be relA dependent $(21,25)$. Our study demonstrated that the memory can be remarkably long-lasting, as 1-h carbon starvation gave an increase in survival for at least $4 \mathrm{~d}$ in the wild-type strain grown in glucose medium. The molecular mechanism underlying this long-term memory is yet to be investigated, but in all likelihood it is linked to the abrupt RelA-mediated rise in (p)ppGpp upon starvation because the starvation-pulse effect on long-term survival was abolished in the $\triangle$ relA mutant. In further support of this hypothesis, there was no long-term effect when glycerol was used as the carbon source, which could be due to the high basal level of (p)ppGpp in glycerol relative to glucose minimal medium (8). The sensitivity of the survival fraction to the rather short perturbation may be consistent with the idea that there is a threshold in some molecule concentration to determine if the cell becomes a persister or not $(31,32)$ because a small perturbation can have a major impact on the occurrence of rare expression patterns that exceed an extreme threshold (33). This observation also alerts us that a small perturbation in the experimental procedure may strongly affect the result of persister assays.

This study shows that the long-term killing of E. coli in ciprofloxacin is not adequately described by biphasic dynamics. At least three phases of killing were present in the data. Thus, despite the emphasis on at least a biphasic behavior to define persistence (4), a third, or even fourth, phase of killing occurs that may even be more clinically relevant. The presence of additional phases also means that the population survival time will be underestimated by predictions from the biphasic killing assumption. Indeed, it is not sufficient to measure killing dynamics for only $5 \mathrm{~h}$ and then extrapolate the population survival time from there.

The detailed molecular mechanism of the observed persistence is not the focus of the current study. Nevertheless, it is worth mentioning that ciprofloxacin has been reported to induce persistence via toxin activation through the SOS response in the killing dynamics up to $6 \mathrm{~h}$ (34). The existence of more than two killing phases indicates that different mechanisms may play roles for longer term persistence on top of the previously studied ones. For the future study of the molecular mechanisms of persistence, attention should be paid to which time scale of the survival the pathway affects.

The population growth rate was found to be positively correlated with the killing rate in the initial phase. However, the correlation was diminished in the longer term survival, and lost in the third phase of killing. In shorter persister assays, a difference in growth rate, such as between different mutants, might strongly confound results when analysing persistence fractions. These differences seem smaller and less relevant in later phases of killing.

This investigation of long-term killing dynamics has added to the concept of bacterial persistence as a complex phenomenon. During long-term antibiotics treatment, different mechanisms could account for bacterial survival on different time scales (an hour, a day, a week), although the (p)ppGpp level at the time of initial exposure to the antibiotic seems to be important in all cases. As such, persistence seems to be a time-dependent phenomenon, where different survival mechanisms account for different bacterial life spans. The presence of several phases in the killing dynamics begs the question if a more extended concept should replace the simplified concept of bacterial persistence. 


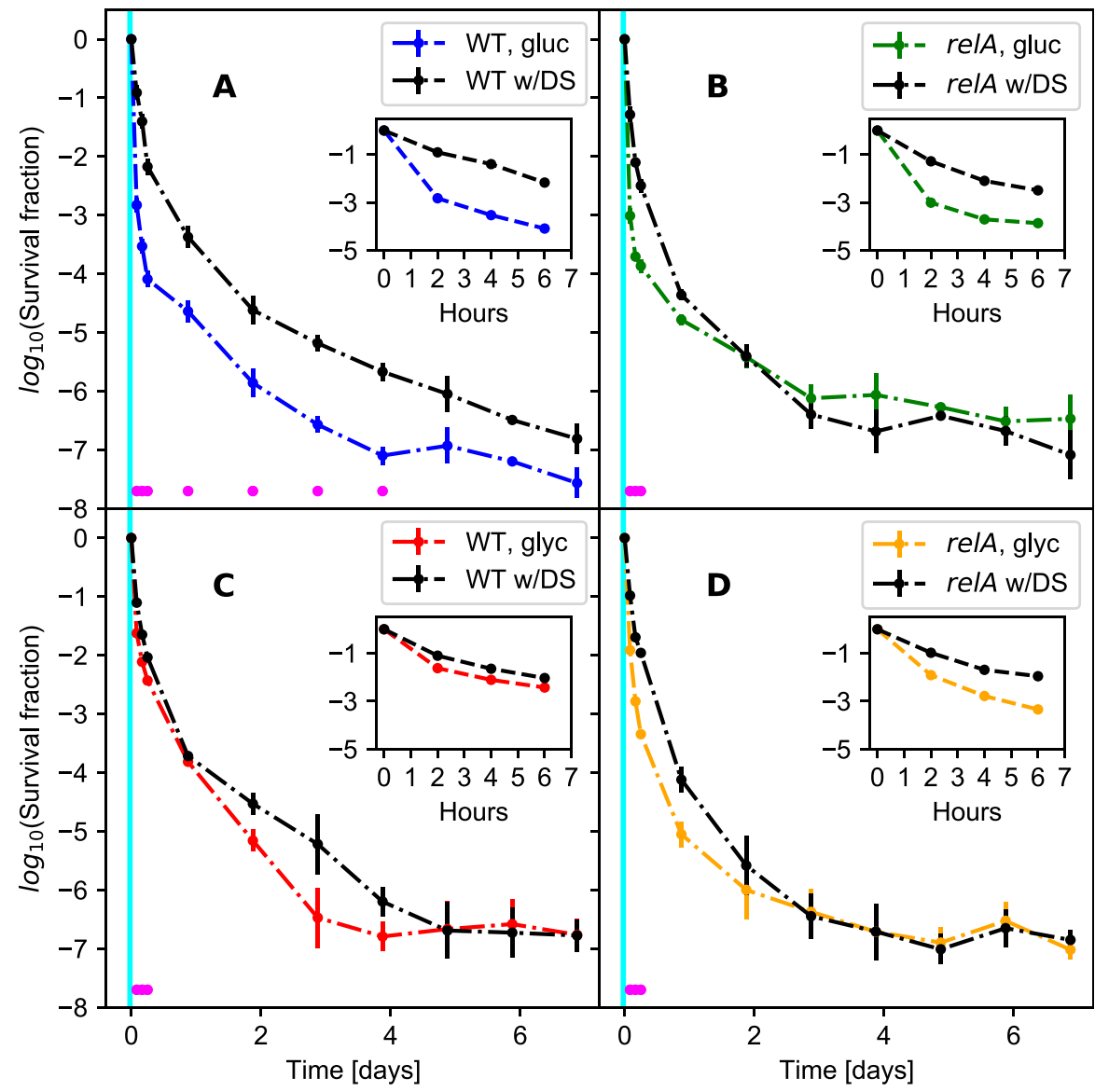

Figure 4. The effect of a short starvation-pulse on the survival dynamics.

All strains and conditions are shown with and without the downshift. The black line is with the downshift, the colored line is without the downshift. The magenta dots represent statistically different data points as tested by an unequal variances two-sided $t$ test. The cyan interval illustrates the downshift period before the killing assay. (A) The wild type in glucose. A downshift in glucose significantly enhances wild-type survival for $4 \mathrm{~d}$ and seems to be increased for up to all $7 \mathrm{~d}$. (B) The $\triangle$ relA mutant in glucose. (C) The wild type in glycerol. (D) The $\triangle$ relA mutant in glycerol.

\section{Materials and Methods}

\section{Strains}

The strain MAS1081 (MG1655 $\mathrm{rph}^{+}$gatC ${ }^{+} g l p R^{+}$) was used as the wild type (35). The $\triangle$ relA, MAS1191, is MAS1081 made $\Delta$ relA251::Kan by P1 transduction from CF1651 (36) followed by selection on kanamycin.

\section{Long-term killing assay}

A single colony of the E. coli strain grown on an agar plate was incubated overnight in the target medium (MOPS minimal medium with either glucose or glycerol as the carbon source (37). See the next subsection for the recipe). This was performed for each biological replicate. The overnight culture was diluted 1:107 in $10 \mathrm{ml}$ target medium in a 100-ml Erlenmeyer flask. The flask was continuously shaken at 160 RPM by a shaker platform (New Brunswick Innova 2300 [orbit $5.1 \mathrm{~cm}$ ], Eppendorf, Germany) in a $37^{\circ} \mathrm{C}$ room. Hours later, the culture was diluted further, at least 1:10², in $100 \mathrm{ml}$ preheated medium in a 1-L Erlenmeyer flask, reaching a total dilution of at least $1: 10^{9}$. The $\mathrm{OD}_{436}$ of the cultures never exceeded 0.3 because of back dilutions to ensure the balanced growth. The growth rates were calculated based on at least five measurements in the measurable $\mathrm{OD}_{436}$ interval from 0.03 to 0.3 (Fig S5 and Table S1). Carbon starvation was introduced by filtration of the culture (typically around $20 \mathrm{ml}$ ), followed by immediate resuspension of the cells on the filter in $40 \mathrm{ml}$ of the preheated target medium (with or without carbon source) in a 300-ml Erlenmeyer flask. As such, both the control and the starved culture were exposed to filtration and resuspension. The medium volume never exceeded 14 percent of the flask volume. The starvation was verified by measuring $\mathrm{OD}_{436}$ twice during the 1-h downshift, to confirm either an increase (control culture) or no change (starving culture) in biomass. The $\mathrm{OD}_{436}$ measurements verified that a starvation took place for all the experiments that were supposed to experience a downshift. It also verified that growth, with a doubling time approximately close to the expected value, took place in the control cultures

After $1 \mathrm{~h}$ of starvation, a sample was taken immediately before ciprofloxacin $(10 \mu \mathrm{g} / \mathrm{ml})$ was added along with the carbon source, which was replenished to end the downshift. Most of the samples were taken at times 2, 4, 6/7, and $21+24 \cdot n$ hours for $n \in[0 ; 6]$ (see next subsection for the detail of the sampling timing). The samples were put on ice for a few minutes and then centrifuged for $10 \mathrm{~min}$ at $4^{\circ} \mathrm{C}$ at $10,000 \mathrm{~g}$. The supernatant was removed, and the cell pellet was resuspended in room temperature MOPS buffer with no supplements. The sample was diluted appropriately, never more than 1:10² per step, corresponding to 10 in $990 \mu \mathrm{l}$. The sample was 
plated with $200 \mu \mathrm{l}$ per plate on minimal medium plates containing the target medium. The plates were kept at $37^{\circ} \mathrm{C}$ for at least $1 \mathrm{wk}$ and all colonies were counted. The whole experiment is illustrated in Fig 3. Detection limit of each experiment is presented in Fig S6.

\section{Correction for difference in sampling times}

Most samples were obtained at approximately hours 2, 4, 6, $21+24 \cdot n$ hours for $n \in[0 ; 6]$. The wild-type glucose experiments were sampled at approximately $2,4,7,21+24 \cdot n$, but one of the experiments in glucose had the following time points $2,4,7,18+24 \cdot n$ where $n \in[0 ; 6]$. We corrected this dataset, by calculating a straight line between the data point of interest and the following data point in log space. We used this straight line to extrapolate the value to the timepoint of interest. The original data values and corrected values are presented in Table S2. All statistical analyses were carried out with and without this correction, to make sure it did not affect the results.

\section{Growth medium}

According to reference 37, MOPS minimal medium was made by mixing the following:

(i) $100 \mathrm{ml} 10 \times$ MOPS medium that contains $40 \mathrm{mM}$ MOPS, $4 \mathrm{mM}$ tricine, $1.32 \mathrm{mM} \mathrm{K}_{2} \mathrm{HPO}_{4}, 9.52 \mathrm{mM} \mathrm{NH}_{4} \mathrm{Cl}, 0.523 \mathrm{mM} \mathrm{MgCl}, 0.276$ $\mathrm{mM} \mathrm{K}_{2} \mathrm{SO}_{4}, 0.01 \mathrm{mM} \mathrm{FeSO}_{4}, 0.5 \mu \mathrm{M} \mathrm{CaCl}_{2}, 50 \mathrm{mM} \mathrm{NaCl}$, and trace metals (see reference 37).

(ii) Carbon source (one of either)

(1) $10 \mathrm{ml} 20 \%$ glucose (for $0.2 \% \mathrm{wt} / \mathrm{vol}$ glucose minimal medium)

(2) $8 \mathrm{ml} 50 \%$ glycerol (for $0.4 \%$ vol/vol glycerol minimal medium). The $50 \%$ glycerol stock solution is stored at $-20^{\circ} \mathrm{C}$.

(iii) Fill up to $1 \mathrm{~L}$ by adding milliQ water

Mops minimal medium plates were made by adding $15 \mathrm{~g}$ Bacto agar (autoclaved with the milliQ water) to the Mops minimal medium recipe above.

\section{Controls}

After each completed experiment ( $7 \mathrm{~d}), 200 \mu \mathrm{l}$ of culture, still containing antibiotics, was spread on a plate with the target medium. This was left at $37^{\circ} \mathrm{C}$ for at least $7 \mathrm{~d}$, and no growth confirmed the absence of a growing resistant culture in the flask. In addition, the activity of the antibiotics in the culture was tested after $7 \mathrm{~d}$ by dropping $20 \mu \mathrm{l}$ on a lawn of growing E. coli.

\section{Data from each biological replicate}

Each biological replicate of the glucose experiments are shown in Fig S7, whereas the replicates of glycerol experiments are shown in Fig S2. If the observed CFU was zero (no colonies counted), the value zero was replaced by the detection limit defined as one colony per plate.

\section{Analysis of time scales in the killing dynamics}

Time scales in the killing dynamics were statistically identified by fitting a sum of exponential functions to the data. The model with the least number of parameters, that could not significantly be rejected, was then chosen (38). The functional form of the models was a sum of exponential functions. The number of exponential functions in the sum corresponded to the number of time scales. A biphasic killing curve would, for example, be well fitted by the sum of two exponentials. The $x^{2}$-test for goodness of fit is used for identifying the appropriate model using the Minuit minimization software $(39,40)$. The functional form of the models are as follows:

$$
\begin{aligned}
& \text { Model1 }\left(t, a_{0}\right)=\exp \left(-a_{0} t\right), \\
& \text { Model2 }\left(t, a_{0}, a_{1}, b_{1}\right)=\left(1-b_{1}\right) \exp \left(-a_{0} t\right)+b_{1} \exp \left(-a_{1} t\right), \\
& \text { Model3 }\left(t, a_{0}, a_{1}, b_{1}, a_{2}, b_{2}\right)=\left(1-b_{1}-b_{2}\right) \exp \left(-a_{0} t\right)+ \\
& b_{1} \exp \left(-a_{1} t\right)+b_{2} \exp \left(-a_{2} t\right), \\
& \text { Model }\left(t, a_{0}, a_{1}, b_{1}, a_{2}, b_{2}, a_{3}, b_{3}\right)=\left(1-b_{1}-b_{2}-b_{3}\right) \\
& \exp \left(-a_{0} t\right)+b_{1} \exp \left(-a_{1} t\right)+b_{2} \exp \left(-a_{2} t\right)+b_{3} \exp \left(-a_{3} t\right) .
\end{aligned}
$$

Each exponential function contains a specific time scale which corresponds to each exponent. A biphasic killing curve would, for example, be well fitted by the sum of two exponentials. To statistically determine which model fits to the data, each model is fitted to the data by using least squares and the Minuit optimization software $(39,40)$. The $x^{2}$ is then calculated for each of these models. The degrees of freedom correspond to the number of data points with the subtraction of the number of parameters for fitting and one degree of freedom for normalizing the data. Thus, the probability that the data do not correspond to the model is calculated using the $x^{2}$ cumulative distribution. The $P$-value was chosen to be 0.05 . For the wild-type strain grown in glycerol, the Model 4 was an appropriate fit, for the three other datasets, the Model 3 was an appropriate fit. The parameters estimated are given in supplement in Table S3. The fits are shown in the supplement in Fig S3.

\section{Display of fits in Fig 1}

The functions shown in Fig 1 all stem from the fit of Model 3 to the data. Model 3 is given by the following equation:

$$
\text { Model3 }=\left(1-b_{1}-b_{2}\right) \exp \left(-a_{0} t\right)+b_{1} \exp \left(-a_{1} t\right)+b_{2} \exp \left(-a_{2} t\right) .
$$

The functions displayed are then given by the three terms of the fit shown separately, as given by:

$$
\begin{aligned}
\text { term } 1 & =\left(1-b_{1}-b_{2}\right) \exp \left(-a_{0} t\right), \\
\text { term } 2 & =b_{1} \exp \left(-a_{1} t\right), \\
\text { term } 3 & =b_{2} \exp \left(-a_{2} t\right) .
\end{aligned}
$$


This is to illustrate the contribution from each exponential time scale.

In addition, the second phase of a biphasic fit to the data shown in the inset, is also shown in the main figure and in the inset.

\section{Statistical analysis}

All killing curves are based on three biological replicates. The mean is determined as the mean of the logarithmic values, which corresponds to the geometric mean. This is done to get a more adequate mean-value representation in log space. The uncertainties are also calculated as the standard deviations of the log-transformed values. Whenever a data point had the value zero, which happened frequently, that value was replaced with the detection limit, to get a sensible value in log space.

An unequal variance two-sided $t$ test was used to determine significant differences between two data points at the same time point $(P<0.05)$

\section{ppGpp measurements}

The measurements were performed essentially as described in reference 41 and used in references 42,43 , and 44 . In short, cultures were grown for two generations in the presence of $75 \mu \mathrm{Ci} / \mathrm{ml} 32 \mathrm{P}$-phosphate at a total phosphate concentration of $0.33 \mathrm{mM}$. At the time of starvation, cultures were filtered, washed in medium without glucose and phosphate and resuspended in the medium without glucose but containing $32 \mathrm{P}$ at the same specific activity as during growth. These steps were performed at $37^{\circ} \mathrm{C}$ and lasted less than $2 \mathrm{~min}$. For determination of the nucleotide pools, $100 \mu \mathrm{l}$ of culture was harvested into $20 \mu \mathrm{l} 2 \mathrm{M}$ formic acid at $0^{\circ} \mathrm{C}$. After centrifugation, the nucleotides in the supernatant were separated by chromatography on polyethyleneimine-cellulose plates. The activities of the individual spots were quantified by Phospholmager scans (Typhoon Phosphor Imager FLA7000 [GE Healthcare]) of the plates. The specific activity of the signal was determined from a medium sample from the individual cultures spotted onto the same brand of plates that were exposed together with the chromatograms.

\section{Supplementary Information}

Supplementary Information is available at https://doi.org/10.26508/lsa. 202101076.

\section{Acknowledgements}

MS Svenningsen and N Mitarai thank S Semsey for fruitful discussions. This work was supported by the Danish National Research Foundation (DNRF120), the Independent Research Fund Denmark (8049-00071B and 8021-00280A), and the Villum foundation (00028054).

\section{Author Contributions}

MS Svenningsen: conceptualization, data curation, formal analysis, investigation, visualization, methodology, and writing-original draft, review, and editing.
SL Svenningsen: conceptualization, resources, supervision, funding acquisition, and writing-review and editing.

MA Sørensen: conceptualization, resources, data curation, formal analysis, supervision, funding acquisition, investigation, visualization, methodology, and writing-review and editing.

N Mitarai: conceptualization, resources, formal analysis, supervision, funding acquisition, investigation, methodology, and writing-original draft, review, and editing.

\section{Conflict of Interest Statement}

The authors declare that they have no conflict of interest.

\section{References}

1. Pezzani MD, Be G, Cattaneo P, Zaffagnini A, Gobbi F, Rodari P, Bisoffi Z, Tacconelli $E$ (2019) Evidence based review on optimal duration of antibiotic therapy for bacterial infections to support antimicrobial stewardship recommendations WHO Secretariat Nicola Magrini, Secretary of the Expert Committee on Selection and Use of Essential Medicines 1-28. https://www.who.int/selection_medicines/committees/ expert/22/applications/ABWG_optimal_duration_AB.pdf

2. Bigger J (1944) Treatment of staphylococcal infections with penicillin by intermittent sterilisation. The Lancet 244: 497-500. doi:10.1016/s01406736(00)74210-3

3. Balaban NQ, Merrin J, Chait R, Kowalik L, Leibler S (2004) Bacterial persistence as a phenotypic switch. Science 305: 1622-1625. doi:10.1126/ science.1099390

4. Balaban NQ, Helaine S, Lewis K, Ackermann M, Aldridge B, Andersson DI, Brynildsen MP, Bumann D, Camilli A, Collins JJ, et al (2019) Definitions and guidelines for research on antibiotic persistence. Nat Rev Microbiol 17 441-448. doi:10.1038/s41579-019-0196-3

5. Harms A, Fino C, Sфrensen MA, Semsey S, Gerdes K (2017) Prophages and growth dynamics confound experimental results with antibiotictolerant persister cells. MBio 8: 1-18. doi:10.1128/mBio.01964-17

6. Brauner A, Fridman O, Gefen O, Balaban NQ (2016) Distinguishing between resistance, tolerance and persistence to antibiotic treatment Nat Rev Microbiol 14: 320-330. doi:10.1038/nrmicro.2016.34

7. Keren I, Kaldalu N, Spoering A, Wang Y, Lewis K (2004) Persister cells and tolerance to antimicrobials. FEMS Microbiol Lett 230: 13-18. doi:10.1016/ S0378-1097(03)00856-5

8. Bremer H, Dennis PP (2008) Modulation of chemical composition and other parameters of the cell at different exponential growth rates, EcoSal Plus 3. doi:10.1128/ecosal.5.2.3

9. Scott M, Gunderson CW, Mateescu EM, Zhang Z, Hwa T (2010) Interdependence of cell growth and gene expression: Origins and consequences. Science 330: 1099-1102. doi:10.1126/science.1192588

10. Tuomanen E, Cozens R, Tosch W, Zak O, Tomasz A (1986) The rate of killing of Escherichia coli by beta-lactam antibiotics is strictly proportional to the rate of bacterial growth. J Gen Microbiol 132: 1297-1304. doi:10.1099/00221287-132-5-1297

11. Lee AJ, Wang S, Meredith HR, Zhuang B, Dai Z, You L (2018) Robust, linear correlations between growth rates and $\beta$-lactam-mediated lysis rates, Proc Natl Acad Sci U S A 115: 4069-4074. doi:10.1073/pnas.1719504115

12. Pontes MH, Groisman EA (2019) Slow growth determines nonheritable antibiotic resistance in Salmonella enterica, Sci Signal 12: 1-11. doi:10.1126/scisignal.aax3938

13. Windels EM, Meriem ZB, Zahir T, Verstrepen KJ, Hersen P, Van den Bergh $B$, Michiels J (2019) Isolation of persisters enabled by $\beta$-lactam-induced 
filamentation reveals their single-cell awakening characteristics. Commun Biol 2: 426. doi:10.1038/s42003-019-0672-3

14. Simsek E, Kim M (2019) Power-law tail in lag time distribution underlies bacterial persistence. Proc Natl Acad Sci U S A 116: 17635-17640. doi:10.1073/pnas.1903836116

15. Goormaghtigh F, Van Melderen L (2019) Single-cell imaging and characterization of Escherichia coli persister cells to ofloxacin in exponential cultures. Sci Adv 5: eaav9462. doi:10.1126/sciadv.aav9462

16. Goormaghtigh F, Fraikin N, Putrinš M, Hallaert T, Hauryliuk V, Garcia-Pino A, Sjödin A, Kasvandik S, Udekwu K, Tenson T, et al (2018) Reassessing the role of type II toxin-antitoxin systems in formation of Escherichia coli type II persister cells. MBio 9: 1-14. doi:10.1128/mBio.00640-18

17. Helaine S, Cheverton AM, Watson KG, Faure LM, Matthews SA, Holden DW (2014) Internalization of salmonella by macrophages induces formation of nonreplicating persisters. Science 343: 204-208. doi:10.1126/ science. 1244705

18. Harms A, Maisonneuve E, Gerdes K (2016) Mechanisms of bacterial persistence during stress and antibiotic exposure. Science 354: 354 doi:10.1126/science.aaf4268

19. Zalis EA, Nuxoll AS, Manuse S, Clair G, Radlinski LC, Conlon BP, Adkins J, Lewis K (2019) Stochastic variation in expression of the tricarboxylic acid cycle produces persister cells. MBio 10: 1-10. doi:10.1128/mBio.01930-19

20. Shan Y, Brown Gandt A, Rowe SE, Deisinger JP, Conlon BP, Lewis K (2017) ATP-dependent persister formation in Escherichia coli. MBio 8: e02267-16. doi:10.1128/mBio.02267-16

21. Svenningsen MS, Veress A, Harms A, Mitarai N, Semsey S (2019) Birth and resuscitation of (p)ppGpp induced antibiotic tolerant persister cells. Sci Rep 9: 6056. doi:10.1038/s41598-019-42403-7

22. Yamasaki R, Song S, Benedik MJ, Wood TK (2019) Persister cells resuscitate using membrane sensors that activate chemotaxis, lower CAMP levels, and revive ribosomes. iscience 23: 100792. doi:10.1016/ j.isci.2019.100792

23. Molina-Quiroz RC, Silva-Valenzuela C, Brewster J, Castro-Nallar E, Levy SB, Camilli A (2018) Cyclic AMP regulates bacterial persistence through repression of the oxidative stress response and SOS-dependent DNA repair in uropathogenic Escherichia coli. MBio 9: e02144-17. doi:10.1128/ mBio.02144-17

24. Nyström T (2004) Stationary-phase physiology. Annu Rev Microbiol 58: 161-181. doi:10.1146/annurev.micro.58.030603.123818

25. Korch SB, Henderson TA, Hill TM (2003) Characterization of the hipA7 allele of Escherichia coli and evidence that high persistence is governed by (p)ppGpp synthesis. Mol Microbiol 50: 1199-1213. doi:10.1046/j.13652958.2003.03779.x

26. Radzikowski JL, Schramke H, Heinemann M (2017) Bacterial persistence from a system-level perspective. Curr Opin Biotechnol 46: 98-105. doi:10.1016/j.copbio.2017.02.012

27. Hui S, Silverman JM, Chen SS, Erickson DW, Basan M, Wang J, Hwa T, Williamson JR (2015) Quantitative proteomic analysis reveals a simple strategy of global resource allocation in bacteria. Mol Syst Biol 11: e784. doi:10.15252/msb.20145697

28. Ryals J, Little R, Bremer H (1982) Control of rRNA and tRNA syntheses in Escherichia coli by guanosine tetraphosphate. J Bacteriol 151: 1261-1268. doi:10.1128/JB.151.3.1261-1268.1982
29. Lazzarini RA, Cashel M, Gallant J (1971) On the regulation of guanosine tetraphosphate levels in stringent and relaxed strains of Escherichia coli. J Biol Chem 246: 4381-4385. doi:10.1016/s0021-9258(18)62023-x

30. Brown DR (2019) Nitrogen starvation induces persister cell formation in Escherichia coli. J Bacteriol 201: e00622-18. doi:10.1128/JB.00622-18

31. Rotem E, Loinger A, Ronin I, Levin-Reisman I, Gabay C, Shoresh N, Biham O, Balaban NQ (2010) Regulation of phenotypic variability by a threshold-based mechanism underlies bacterial persistence. Proc Natl Acad Sci U S A 107: 12541-12546. doi:10.1073/pnas.1004333107

32. Michiels JE, Van den Bergh B, Verstraeten N, Michiels J (2016) Molecular mechanisms and clinical implications of bacterial persistence. Drug Resist Updat 29: 76-89. doi:10.1016/j.drup.2016.10.002

33. Svenningsen MS, Semsey S, Mitarai N (2019) Gene expression changes with minor effects on the population average have major effects on the occurrence of cells with extreme protein concentrations. mSphere 4 : 1-6. doi:10.1128/mSphere.00575-18

34. Dörr T, Vulic M, Lewis K (2010) Ciprofloxacin causes persister formation by inducing the TisB toxin in Escherichia coli. PLoS Biol 8: e1000317. doi:10.1371/journal.pbio.1000317

35. Gummesson B, Shah SA, Borum AS, Fessler M, Mitarai N, Sørensen MA Svenningsen SL (2020) Valine-Induced isoleucine starvation in Escherichia coli K-12 studied by spike-in normalized RNA sequencing. Front Genet 11: 144. doi:10.3389/fgene.2020.00144

36. Xiao H, Kalman M, Ikehara K, Zemel S, Glaser G, Cashel M (1991) Residual guanosine 3',5'-bispyrophosphate synthetic activity of relA null mutants can be eliminated by spoT null mutations. J Biol Chem 266: 5980-5990. doi:10.1016/s0021-9258(19)67694-5

37. Neidhardt FC, Bloch PL, Smith DF (1974) Culture medium for enterobacteria. J Bacteriol 119: 736-747. doi:10.1128/JB.119.3.736-747.1974

38. Barlow RJ (1993) Statistics: A Guide to the Use of Statistical Methods in the Physical Science. Chichester, UK: John Wiley \& Sons.

39. James F, Roos M (1975) Minuit: A system for function minimization and analysis of the parameter errors and correlations. Computer Phys Commun 10: 343-367. doi:10.1016/0010-4655(75)90039-9

40. Iminuit: A Python interface to Minuit (2020)

41. Sørensen MA, Jensen KF, Pedersen S (1994) High concentrations of ppGpp decrease the RNA chain growth rate. Implications for protein synthesis and translational fidelity during amino acid starvation in Escherichia coli. J Mol Biol 236: 441-454. doi:10.1006/jmbi.1994.1156

42. Sinha AK, Winther KS (2021) The RelA hydrolase domain acts as a molecular switch for (p)ppgpp synthesis. Commun Biol 4: 434-510. doi:10.1038/s42003-021-01963-z

43. Tian C, Roghanian M, Jørgensen MG, Sneppen K, Sørensen MA, Gerdes K Mitarai N (2016) Rapid curtailing of the stringent response by toxinantitoxin module-encoded mRNases. J Bacteriol 198: 1918-1926. doi:10.1128/JB.00062-16

44. Sarubbi E, Rudd KE, Xiao H, Ikehara K, Kalman M, Cashel M (1989) Characterization of the spoT gene of Escherichia coli. J Biol Chem 264: 15074-15082. doi:10.1016/s0021-9258(18)63813-x

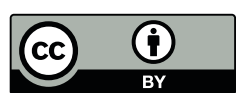

License: This article is available under a Creative Commons License (Attribution 4.0 International, as described at https://creativecommons.org/ licenses/by/4.0/). 